\title{
Interação entre abelha Trigona hyalinata (Lepeletier, 1836) (Hymenoptera: Apidae) e Aethalion reticulatum Linnaeus, 1767 (Hemiptera: Aethalionidae) em Clitoria fairchildiana Howard (Papilionoideae)
}

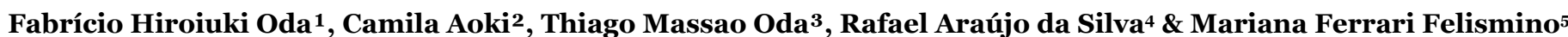

1. Universidade Federal de Goiás, Laboratório de Comportamento Animal, Instituto de Ciências Biológicas, Campus Samambaia, e-mail: fabricio oda@hotmail.com, Autor para correspondência. 2.Universidade Federal de Mato Grosso do Sul. Programa de Pós-Graduação em Ecologia e Conservação, e-mail: aokicamila@yahoo.com.br. 3. Especialização em Gestão Ambiental pelas Faculdades Integradas de Paranaíba - FIPAR, email: thiago oda@hotmail.com. 4. Graduado em Engenharia Florestal pela Universidade Estadual do Centro-Oeste - UNICENTRO, e-mail: rafaflorestal@hotmail.com. 5. Universidade Estadual de Maringá - UEM, Departamento de Biologia Celular e Genética, e-mail: mariferrari 82@hotmail.com.

EntomoBrasilis 2 (2): 58-6o (2009)

Resumo. A cigarrinha do pedúnculo Aethalion reticulatum (Linnaeus, 1767) é um inseto que se alimenta da seiva de várias espécies vegetais e pode viver em simbiose com formigas do gênero Camponotus (Hymenoptera: Formicidae, Formicinae), atacando folhas e ramos de inúmeras plantas cultivadas e nativas, tais como acácia-negra, algodoeiro, aroeira, cafeeiro, eucalipto, citros, etc. Algumas relações aparentemente comensais podem ocorrer entre cigarrinhas e abelhas sem ferrão, principalmente Trigona. No presente estudo, observamos a interação entre a abelha irapuá, Trigona hyalinata (Lepeletier, 1836) e a cigarrinha do pedúnculo, A. reticulatum em Clitoria fairchildiana Howard (sombreiro). A interação entre T. hyalinata e A. reticulatum foi registrada por meio de fotografias digitais. A estimulação da cigarrinha para a liberação de exsudato iniciava-se quando as abelhas pousavam sobre as colônias de ninfas e adultos de $A$. reticulatum e estimulavam os indivíduos andando por cima deles, principalmente da região anterior (cabeça) para a posterior (abdome) do corpo da cigarrinha. Posteriormente, as abelhas tocavam as antenas na parte distal do abdome estimulando a secreção de exsudato e prontamente sugavam essa substância. Esta interação é mutuamente benéfica, sem implicar necessariamente dependência ou interdependência obrigatória, e pode promover uma proteção contra os inimigos naturais da cigarrinha. Todavia, a ocorrência de uma alta infestação de cigarrinhas, associada à intensa atividade de abelhas solicitando honeydew pode levar a depleção de nutrientes da planta, afetando o desenvolvimento e/ou reprodução de C. fairchildiana.

Palavras-Chave: Cigarrinha do Pedúnculo, Honeydew, Irapuá, Mutualismo, Sombreiro.

Interaction between Trigona hyalinata (Lepeletier, 1836) (Hymenoptera: Apidae) and Aethalion reticulatum Linnaeus, 1767 (Hemiptera: Aethalionidae) in Clitoria fairchildiana Howard

(Papilionoideae)

Abstract. The Aethalion reticulatum (Linnaeus, 1767) is an insect that feeds of the sap of several vegetal species and can live in symbiosis with ants from the genera Camponotus (Hymenoptera: Formicidae, Formicinae), attacking leaves and branches of many cultivated and native plants such as acacia-negra, cotton, aroeira, coffee, eucalyptus, citrus, etc. Some apparently commensal relationships can occur between leafhoppers and stingless bees, especially Trigona. In the present study we have observed the interaction between the irapuá bee Trigona hyalinata (Lepeletier, 1836) and the leafhopper of the stalk A. reticulatum in Clitoria fairchildiana Howard (sombreiro). The interaction between T. hyalinata and A. reticulatum was registered through digital photographs. The stimulation of the leafhopper for the release of the honeydew initiated when bees landed on the nymph's and adult's colony of $A$. reticulatum and stimulated the individuals by walking over them, mainly from the anterior region (head) to the posterior one (abdomen) of the leafhopper's body. After that the bees touched their antennas at the distal part of the abdomen stimulating the production of the honeydew and readily sucked the substance. This interaction is mutually beneficial without necessarily implying obligate dependence or interdependence, and can promote a protection against leafhopper natural enemies. Nevertheless, the occurrence of a high leafhopper infestation associated to the intense activity of bees requesting honeydew may leave to depletion of the plant's nutrients, affecting the development and/or of C. fairchildiana.

Key words: Honeydew, Irapuá, Leafhopper of the Stalk, Mutualism, Sombreiro.

A espécie Aethalion reticulatum (Linnaeus) (Hemiptera: Aethalionidae), conhecida como cigarrinha das fruteiras ou cigarrinha do pedúnculo, é um inseto sugador, cujos adultos medem em torno de $10 \mathrm{~mm}$ de comprimento, de cor marrom ferrugínea, com venação das asas salientes e esverdeadas. As fêmeas podem colocar até 100 ovos nos ramos das plantas hospedeiras. Os ovos demoram cerca de 30 dias para eclodirem; durante este tempo, a fêmea permanece junto à postura, protegendo-a. As ninfas possuem o corpo de coloração cinza com estrias amarelas. Permanecem nesta fase durante 45 dias. O ciclo total se completa em 110 dias, com três gerações ao ano (SANTANA et al. 2005). Tanto adultos quanto ninfas sugam a seiva da planta, prejudicando o desenvolvimento de frutos e brotações, podendo, em altas infestações, matar a planta (GALLO 
et al. 2002). Os adultos vivem cerca de dois meses (VIEIRA et al. 2007).

A abelha irapuá, Trigona hyalinata (Lepeletier) (Hymenoptera: Apidae, Meliponinae), é um forrageador generalista (BARBOLA et al. 2000) e ocorre nos estados de Minas Gerais, Bahia, Goiás e São Paulo (SILVEIRA et al. 2002). Clitoria fairchildiana Howard (Leguminosae, Papilionoideae) é conhecida como sombreiro. Sua distribuição concentra-se principalmente na Floresta Ombrófila Densa na Amazônia, em formações secundárias e apresenta nítida preferência por solos férteis e úmidos. Como uma espécie rústica e de rápido crescimento, é extremamente útil nos reflorestamentos heterogêneos destinados à reconstituição da vegetação (LORENZI 1992), sendo comumente utilizados na arborização urbana de diversos estados brasileiros (GUAJARÁ et al. 2003).

A cigarrinha do pedúnculo é um inseto que se alimenta da seiva de várias espécies vegetais e pode viver em simbiose com formigas do gênero Camponotus (Hymenoptera: Formicidae, Formicinae), atacando folhas e ramos de inúmeras plantas cultivadas e nativas, tais como acácia-negra, algodoeiro, aroeira, cafeeiro, eucalipto, citros, etc. (SANTANA et al. 2005). Algumas relações aparentemente comensais podem ocorrer entre cigarrinhas e abelhas sem ferrão, principalmente Trigona. Estas abelhas coletam as secreções açucaradas (honeydew) ricas em nutrientes e liberadas, espontaneamente ou por solicitação, pelas cigarrinhas (relação entendida como trofobiose). As abelhas devem se beneficiar do uso do exsudato (honeydew), mas elas parecem não defender de forma efetiva as cigarrinhas, diferentemente do que ocorre quando estas se associam às formigas (SALT 1929, WILSON 1971, CASTRO 1975).

No presente estudo, observamos a interação entre a abelha irapuá, T. hyalinata e a cigarrinha do pedúnculo, $A$. reticulatum em C. fairchildiana (sombreiro).

Em dezembro de 2007, na área rural do município de Araçatuba, estado de São Paulo, Brasil ( $21^{\circ} 12$ 'S, $\left.50^{\circ} 23^{\prime} \mathrm{W}\right)$, observou-se a presença de colônias de cigarrinhas em ramos de C. fairchildiana. Junto às colônias de cigarrinha observamos a ocorrência de espécimes de T. hyalinata.

O estudo foi conduzido no período de 23 a 28 de dezembro de 2007 em plantas cultivadas no jardim residencial da sede da Estância Santa Rita de Cássia. A interação entre as abelhas e as cigarrinhas foi registrada por meio de observações diárias, iniciadas às 9:00 $\mathrm{h}$ da manhã com término às 17:00 $\mathrm{h}$ da tarde. A escolha do período de observações foi baseada em dados de atividade de vôo de T. hyalinata (CONTRERA et al. 2004). O comportamento dos animais observados foi registrado por meio de fotografias digitais em câmera digital Sony R1.

Observamos espécimes de $T$. hyalinata coletando exsudato de cigarrinhas nos ramos de $C$. fairchildiana. O comportamento observado no presente estudo corrobora o comportamento descrito para $T$. spinipes, em manga, Mangifera indica L. (Anacardiaceae) (VIERA et al. 2007). As abelhas pousavam sobre as colônias de ninfas e adultos de A. reticulatum e estimulavam os indivíduos andando por cima deles, principalmente da região anterior (cabeça) para a posterior (abdome) do corpo da cigarrinha.

A estimulação da cigarrinha para a liberação de exsudato iniciava-se quando as abelhas tocavam suas antenas na cabeça dos indivíduos, descendo até o abdome. Em seguida, as abelhas tocavam na parte posterior do abdome de $A$. reticulatum utilizando os dois primeiros pares de pernas. Posteriormente, as abelhas tocavam as antenas na parte distal do abdome estimulando a secreção de exsudato e prontamente sugavam essa substância (Figura 1).

Não foi observada a presença de formigas do gênero Camponotus junto às colônias de cigarrinhas. Entretanto, comportamento agonístico foi apresentado pelas abelhas para com outras abelhas da mesma espécie durante a interação com A. reticulatum. As abelhas levantavam os dois primeiros pares de pernas, juntamente com as asas, ameaçando o intruso. Esse comportamento foi registrado para $T$. spinipes em $M$. indica, em resposta a presença de abelhas da mesma espécie e formigas do gênero Camponotus (VIEIRA et al. 2007), e está relacionado ao comportamento de defesa do alimento e marcação do território

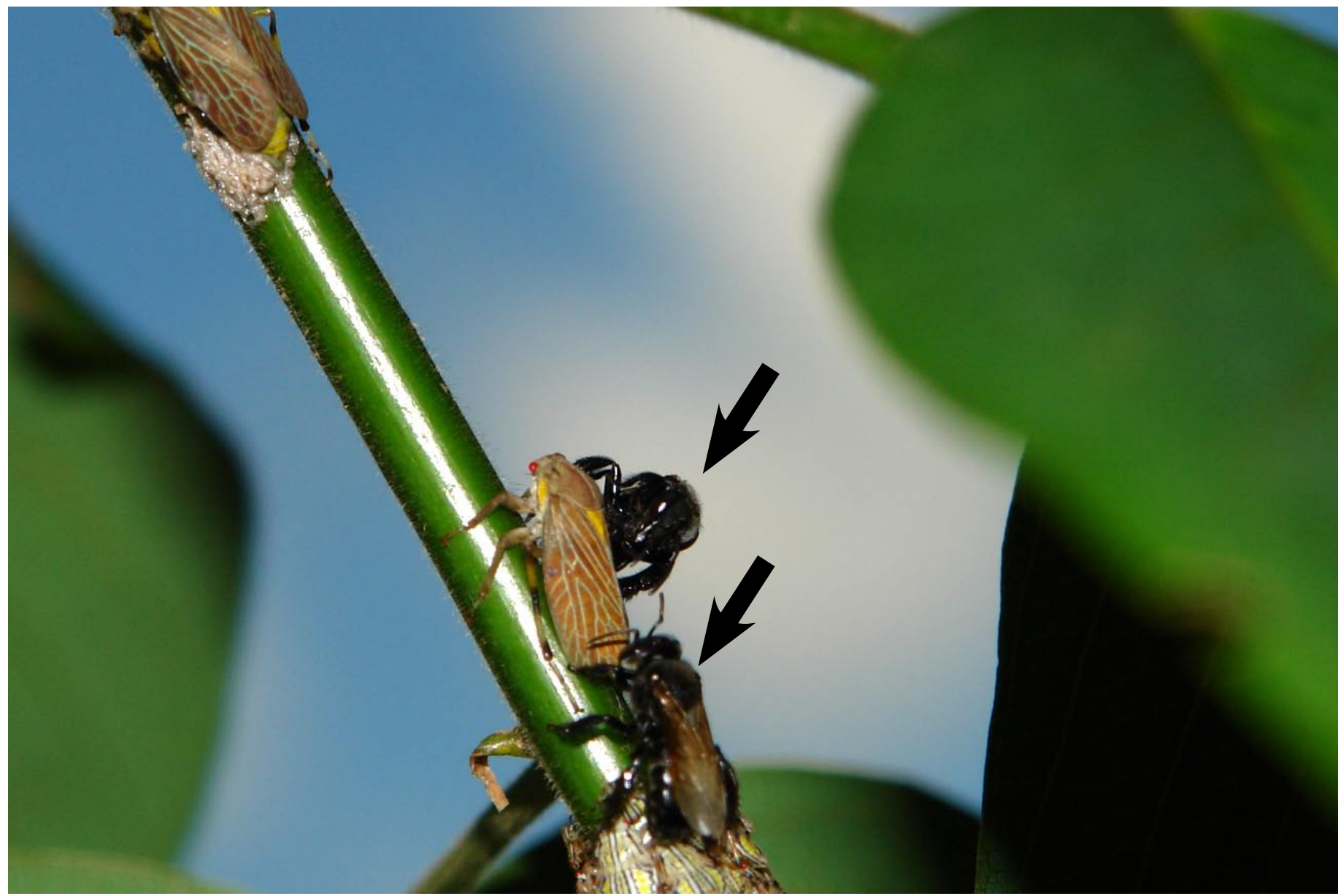

Figura 1. Trigona hyalinata (seta) coletando exsudado (honeydew) de A. reticulatum em pedúnculo de C. fairchildiana. 
(mutualismo defensivo) (RICKFLES 2003).

Aabelha T.hyalinata estabelece com essa cigarrinhauma associação mutuamente benéfica, sem implicar necessariamente dependência ou interdependência obrigatória. Esse mutualismo pode promover uma proteção contra os inimigos naturais da cigarrinha, sendo que as abelhas utilizam a secreção rica em carboidratos para suprir parte de sua dieta alimentar (WAY 1963). LAROCA \& SAKAKIBARA (1976) também observaram esse tipo de interação, todavia, entre Trigona hyalinata branneri e Aconophora flavipes (Germar) (Membracidae).

Outros estudos também registram esse tipo de interação, entretanto, para T. spinipes em associação com A. reticulatum e outras espécies de hemípteros. CASTRO (1975) observou o mutualismo entre $T$. spinipes e $A$. reticulatum em Cajanus indicus Spreng. na presença de Camponotus sp. VIEIRA et al. (2007) observaram a interação entre T. spinipes e A. reticulatum em M. indica. AZEVEDO et al. (2008) observou T. spinipes coletando exsudato de 15 espécies de Membracidae em Cajanus cajan (L.) Millspaugh.

A ocorrência de uma alta infestação de $A$. reticulatum, associada à intensa atividade de abelhas solicitando exsudato pode ocasionar prejuízo ao sombreiro quanto à produção de flores e frutos, pois a constante alimentação pode levar a depleção de nutrientes para planta, afetando o seu desenvolvimento e/ou reprodução (veja VIEIRA et al. 2007).

\section{AGRADECIMENTOS}

Agradecemos a Geralda de P. S. Artioli e Carlos A. Artioli pela permissão concedida para realizarmos o estudo na propriedade da Estância Santa Rita de Cássia. Ao Profo Dr. João M. F. Camargo pela identificação das abelhas. A Mayra Pimenta pelas críticas e sugestões ao manuscrito. A Diego H. Oda pela assistência em campo.

\section{REFERÊNCIAS}

Azevedo, R.L., C.A.L. Carvalho \& O.M. Marques. 2008. Insetos associados à cultura do feijão guandu na região do Recôncavo da Bahia, Brasil. Revista Caatinga, 21: 83-88.

Barbola, I.F., S. Laroca \& M.C. Almeida. 200o. Utilização de recursos florais por abelhas silvestres (Hymenoptera, Apoidea) da Floresta Estadual Passa Dois (Lapa, Paraná, Brasil). Revista Brasileira de Entomologia, São Paulo, 44: 919.

Castro, P.R.C. 1975. Mutualismo entre Trigona spinipes (Fabricius, 1793) e Aethalion reticulatum (L., 1767) em Cajanus indicus Spreng. na presença de Camponotus sp. Ciência e Cultura, 27: 537-539.

\section{Como citar este artigo:}

F.H. Oda, C. Aoki, T.M. Oda, R.A. Silva \& M.F. Felismino, 2009. Interação entre abelha Trigona hyalinata (Lepeletier, 1836) (Hymenoptera: Apidae) e Aethalion reticulatum Linnaeus, 1767 (Hemiptera: Aethalionidae) em Clitoria fairchildiana Howard (Papilionoideae). EntomoBrasilis, 2(2): 58-60. www.periodico.ebras.bio.br/ojs
Contrera, F.A.L., V.L. Imperatriz-Fonseca, \& J.C. Nieh, 2004. Temporal and climatological influences on flight activity in the stingless bee Trigona hyalinata (Apidae, Meliponini). Revista de Tecnologia e Ambiente, Criciúma, 10: 35-43.

Gallo, D., O. Nakano, S. Silveira Neto, R.P.L. Carvalho, G.C. Batista, E. Berti Filho, J.R.P. Parra, R.A. Zucchi, S.B. Alves, J.D. Vendramim, L.C. Marchini, J.R.S. Lopes \& C. Omoto. 2002. Entomologia Agrícola. Piracicaba: Livroceres, 920p.

Guajará, M., A.G. Carvalho, W. Santos, \& K. Gonçalves. 2003. Aspectos da biologia de Euphalerus clitoriae Burckhardt \& Guajará, 2000 (Hemiptera: Psyllidae) sob condições de campo. Floresta e Ambiente, 10: 69-75.

Laroca, S. \& A.M. Sakakibara. 1976. Mutualismo entre Trigona hyalinata branneri (Apidae) e Aconophora flavipes (Membracidae). Revista Brasileira de Entomologia, 20: 7172.

Lorenzi, H. 1992. Árvores Brasileiras: Manual de identificação e cultivo de plantas arbóreas nativas do Brasil. Nova Odessa, Ed. Plantarum, 168p.

Rickfles, R.E. 2003. A Economia da Natureza. $5^{\text {a }}$ Ed. Rio de Janeiro, Ed. Guanabara Koogan, 503p.

Salt, G.A. 1929. Contribution to the ethology of the Meliponinae. Transactions of the Entomological Society of London, 77: 431-470.

Santana, D.L.Q., C.A. Ferreira, E.G. Martins \& H.D. Silva. 2005. Ocorrência de Aethalion reticulatum (Linnaeus, 1767) (Hemiptera: Aethalionidae) em Grevillea robusta. Boletim de Pesquisa Florestal, Colombo, 50: 109-115.

Silveira, F.A., G.A.R. Melo \& E.A.B. Almeida. 2002. Abelhas brasileiras. Sistemática e Identificação. Belo Horizonte, Fundação Araucária, 253p.

Vieira, C.U., C.M. Rodovalho, L.O. Almeida, A.C.S. Siquieroli \& A.M. Bonetti, 2007. Interação entre Trigona spinipes Fabricius, 1793 (Hymenoptera: Apidae) e Aethalion reticulatum Linnaeus, 1767 (Hemiptera: Aethalionidae) em Mangifera indica (Anacardiaceae). Bioscience Journal, Uberlândia, Supplement 1, 23: 10-13.

Way, M.J. 1963. Mutualism between ants and honeydewproducing Homoptera. Annual Review of Entomology, 8: 307-344.

Wilson, E.O. 1971. The insect societies. Cambridge, Harvard University Press, 548p.

Recebido em: 16/02/2009

Aceito em: 20/04/2009

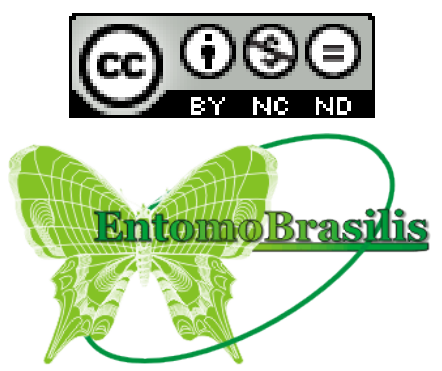

